\title{
A numerical investigation of deployable drag surfaces used for recovery systems
}

\author{
S. Abdolahi \& M. Ebrahimi \\ Aerospace Research Institute, Iran
}

\begin{abstract}
In this research, an investigation into the aerodynamic characteristics of a body with deployable drag surfaces for recovery system has been carried out using computational fluid dynamics. Two models of the body with retracted position of drag surfaces and deployed position of drag surfaces has been considered for studying the influence of drag surfaces on the flow structure and aerodynamic forces. For this purpose force measurement and flow visualization for each case has been carried out in Mach numbers 0.4 and 1.5. Validation of the results has been done through comparing aerodynamic coefficients with results of a semiexperimental method. A general study of the main aerodynamic coefficients shows that at all angles of attack, the coefficient of lift decreases and the coefficient of drag increases. Visualization of the flow structure shows a region of separated flow upstream and a dead flow region with large vortices downstream of the drag surfaces.
\end{abstract}

Keywords: numerical simulation, aerodynamic characteristics, recovery system, drags coefficient, pressure distribution, shock wave, vortex.

\section{Introduction}

Several methods have been employed for recovery of flying objects with various degrees of success. But the most prominent method, especially for heavier bodies is parachute recovery [1], in which a number of parachutes are deployed in a predefined sequence to reduce the body's rate of descent. Physics of supersonic flow around parachutes has its own complexities such as area oscillation and shock wave formation [2]. The controlled deceleration of a flying body is a vital part of many aerospace missions that involves significant technological challenges and creative engineering solutions [3]. For many flying objects, 
parachutes cannot be relied on as the only means of deceleration and recovery because of their weight and altitude. Numerous studies in the past fifty years demonstrate the benefit of developing a new decelerator technology capable of operating at higher Mach numbers and higher dynamic pressures than existing decelerators allow [4].

An effective recovery system must meet three principal criteria: It must be able to reduce body's rate of descent down to the acceptable level, it must not impose unacceptable impact and shock loads to the body when its components are deployed, and finally it must have minimum sensitivity to crosswinds to prevent excessive drift.

For a flying object, which weighs approximately $100 \mathrm{~kg}$, and is expected to reach a maximum altitude of $100 \mathrm{~km}$, trajectory simulation shows that deploying parachutes shortly after re-entry could cause the flying object to drift beyond limits of the test range, due to strong crosswinds in higher layers of atmosphere. Trajectory simulations also show that deploying parachutes at lower levels, where the flying object reaches supersonic speeds, would expose it to impact loads beyond its established structural limit of $18 \mathrm{~g}$ [5].

In this case, use of rigid aerodynamic decelerators such as drag plates and disk gap bands is a solution for reducing body velocity to low-subsonic before deploying parachutes [6]. A drag plate, is a circular plate with an area less than or equal to the area of the base, which is normally connected to the body by a number of belts and deploys after the body starts to descend from maximum altitude [7].

Typical drag plates have a number of inherent shortcomings that have prevented them so far from acquiring prominence among different techniques of aerodynamic deceleration. Although a drag plate can have relatively large drag coefficients in certain flight regimes, its drag generation capability is limited because its effective area cannot be larger than the body's base area. The other problem of the drag plate is that its connection to the flying object is flexible, and the number of connecting belts in this flexible connection is a concern. If a small number of belts are used, they would allow the plate, which is subject to the body's wake, to oscillate, and even become unstable. On the other hand, larger numbers of belts would add to the system's weight and complexity. In some applications, design of hinges which connect the belts to the plate has also been a challenge for the designers [7].

The mentioned shortcomings of drag plates and enhancing effectiveness and performance of the recovery system led to development of alternative method to use of rigid aerodynamic decelerator. The main concept for this method is taken from grid fins [8] and other local protuberances [9] that can be used as either aerodynamic stabilizer or a control surface. The rigid aerodynamic deceleration system presented herein is deployable drag surface folded on the body and released on flight computer's command. The presented design is the first application of this method to reduce body's speed to subsonic range before deploying parachute by increasing drag.

In this paper to study performance of using deployable drag surfaces and the influences of them on body aerodynamic characteristics, three dimensional 
supersonic and subsonic turbulent flows around the body were numerically investigated. The computation of the flow field around such complex configuration, as drag surfaces adjacent fins or wings, is of considerable interest for an accurate calculation and prediction of the shock structures because there is an interaction between drag surfaces shock waves with fins or wings. Accurate determination of forces is required for the calculation of trajectory and structural analysis of body in design and operation.

In these simulations the main focus has been on aerodynamic characteristics. To demonstrate the efficiency and accuracy of the present methodology the aerodynamic characteristics of this configuration including drag and lift forces obtained by commercial CFD code for subsonic and supersonic free stream Mach numbers were compared with the semi-experimental measurements at various angles of attack.

\section{Computational methodologies}

This study is undertaken using Fluent software as a tool to predict the flow field around the body. To investigate the flow field, the Navier-Stokes equations are modelled by using density-based method. The flow considered here is threedimensional, stationary, viscous, and turbulent in which the RNG K-epsilon model has been used for turbulence modelling. To accommodate accurate turbulence modelling, the standard wall functions are selected. A first order accurate method is computed to establish the flow. A second order accurate method is computed to achieve the convergence of the solution.

The verification of the CFD method included quantitative comparisons provided by Missile DATCOM as well as qualitative study of flow structure. Missile DATCOM is a semi-experimental aerodynamic prediction code that calculates aerodynamic forces, moments and stability derivatives as function of angle of attack and Mach number for a variety of axisymmetric and nonaxisymmetric missile configurations.

\subsection{Geometry and boundary conditions}

In this study to find out the influence of the drag surfaces on the aerodynamic characteristic of the body two cases have been considered. Figure 1 shows both the models.

In first case, a body-fins configuration is examined with 2350 in length and 338 in diameter. The configuration has an ogive nose with four tail fins arranged in a cruciform pattern. The fins have a supersonic airfoil cross section. The set of the fins increases the lift force and stability of the body.

In the second case, four drag surfaces deployed in between of each pair of the fins is modelled which placed across the centerline of the vehicle partially block the flow. The drag surfaces are conformed to the outer surface of the body when are retracted. In other words the deployable drag surfaces folded on the body. When the deployment command is issued by the flight computer, the drag 


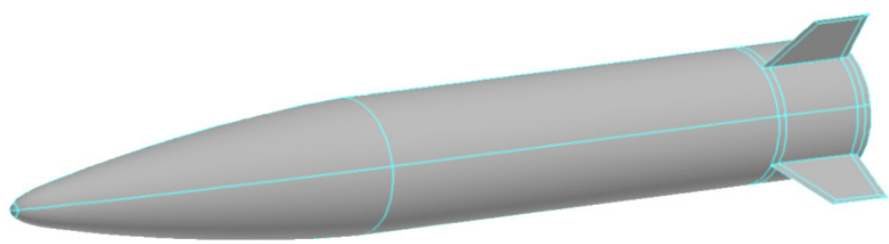

(a)

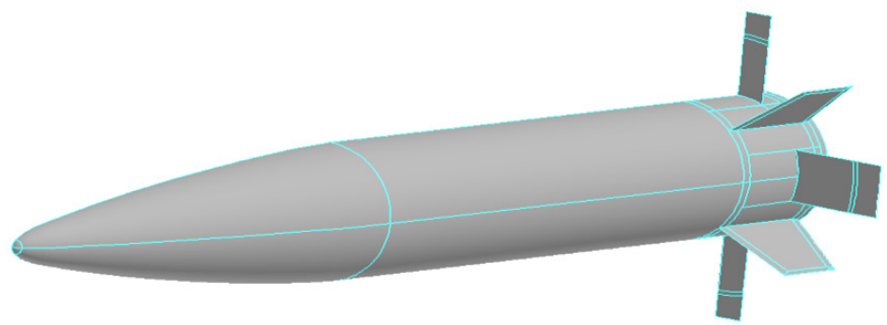

(b)

Figure 1: Geometry of body a) retracted position of drag surfaces b) deployed position of drag surfaces.

surfaces are released, and travel from the retracted position (figure 1a) to the deployed position (figure 1b). This unconventional method has been applied to enhance the drag forces. In this research a half of the model has been considered because of the symmetric flow and geometry to reduce calculation time. Three types of boundary conditions were used: wall, pressure far-field, symmetry.

\subsection{Grid generation}

The accuracy of the CFD results greatly depends on how accurately the configuration is modelled and meshed. The entire solution domain is discretized by a structured grid. Several grid studies were performed to ensure that the grid was fine enough to capture the physical characteristics of the flow. It had been seen that the calculated CA approaches the constant value by increasing the grid qualification. The limitation in computer memory storage is a major factor in preventing further increase in grid size.

The total number of cells is about 4,000,000 for body-fins configuration and 4,500,000 cells for body-fins with deployable drag surfaces. Figure 2 shows the quality of the structured grid adjacent to the surface of the body, nose, fins and drag surfaces. For evaluating the quality of the structured grid adjacent to the body surface, the values of $\mathrm{Y}+$ at the first of wall nodes were calculated. It was shown that most of them have the values more than 30 except for a negligible number of the grids. A trade off between computation time and quality of result led to a grid with finer mesh near to solid surface and coarser mesh adjacent to far field boundaries. 


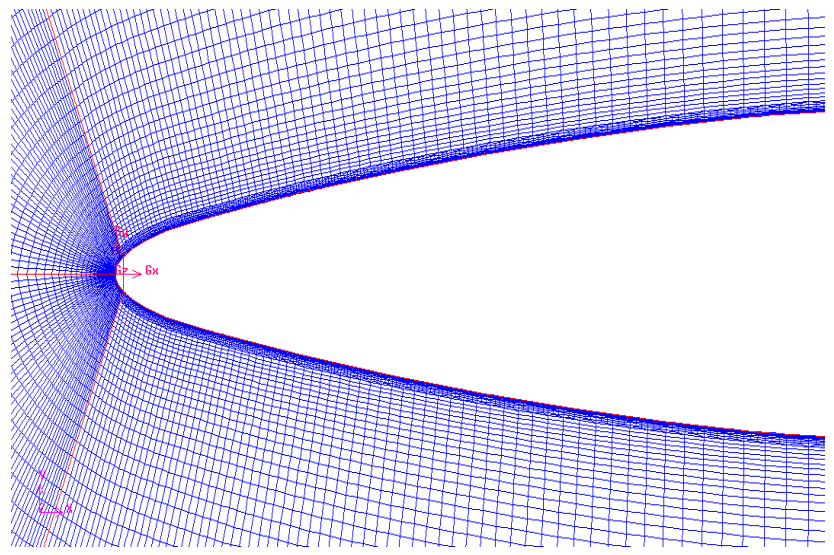

(a)

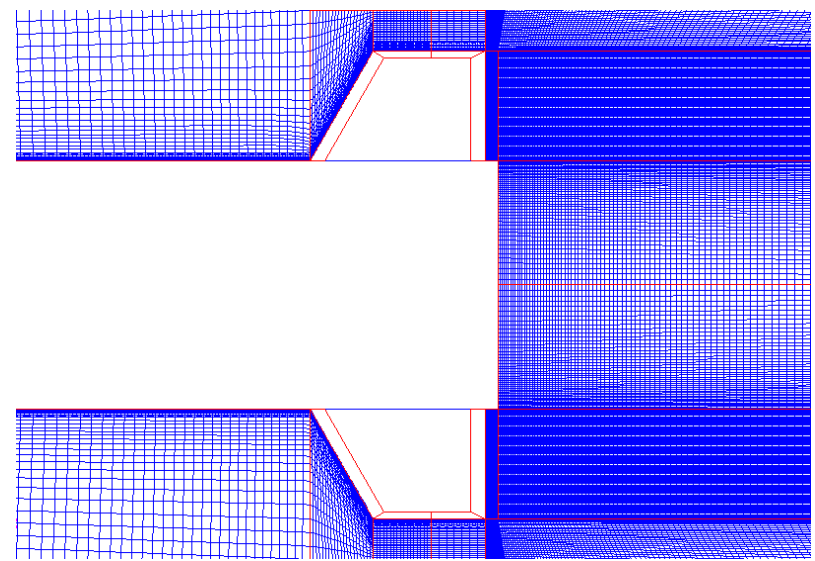

(b)

Figure 2: The quality of the structured grid (a) adjacent to the surface of the nose (b) adjacent to the surface of the body, fins and drag surfaces.

\section{Results and discussion}

A discussion is mainly presented herein to find out the influence of the drag surfaces on the aerodynamic characteristic of the body. A secondary interest in the investigation is the interaction of a drag plates with a stabilizing fins and the effects on the efficiency of stabilizing fins on vehicle in supersonic and subsonic flow. For this purpose force measurement and flow visualization of the numerical flow simulation for each case has been done. Validation of the results has been done through comparing forces with results of semi-experimental method. 


\subsection{Force coefficients}

Body-fins configuration has been considered to study as the first case. The study has been done for subsonic Mach number 0.4 and supersonic Mach number 1.5 at different angles of attack. Semi-experimental method (MD code) is used as aerodynamic prediction code to determine the accuracy of numerical simulation in this paper. Figure 3 shows a comparison between predictions for drag force coefficients for numerical code and semi-experimental method for body before releasing drag surfaces. It is evident that especially at these low angles of attack there is an excellent consistency between solvers. There is a little difference in Mach number 1.5.

As the second case, the configuration of the body-fins with drag surfaces as figure $1 \mathrm{~b}$ has been considered. In this case semi-experimental method is used as aerodynamic prediction codes to determine the accuracy of numerical simulation too with this difference that the drag surfaces are modelled as local protuberance and their effects has been considered on axial force. Therefore the axial force coefficient is only reported for this case in figure 4 . This figure shows that the results from numerical modelling agree well with the trend and almost the magnitude of semi-experimental data.

\subsection{Flow visualization and path line analysis}

Figure 5 is a picture of contour of Mach number from side view of body after releasing drag surfaces at 0 degree angle of attack and Mach number 1.5.The nose oblique shock and expansion waves are clearly visible in this figure. There is a flow separation upstream of the drag surfaces, which causes a thickening of the boundary layer. The thickening leads to compression of the flow and eventually a shock wave. Supersonic flow faces the drag surfaces causing a second shock wave. The secondary shock structure is bowed in nature. Decreasing velocity of cross flow results region of recirculation in flow which produces a separation upstream

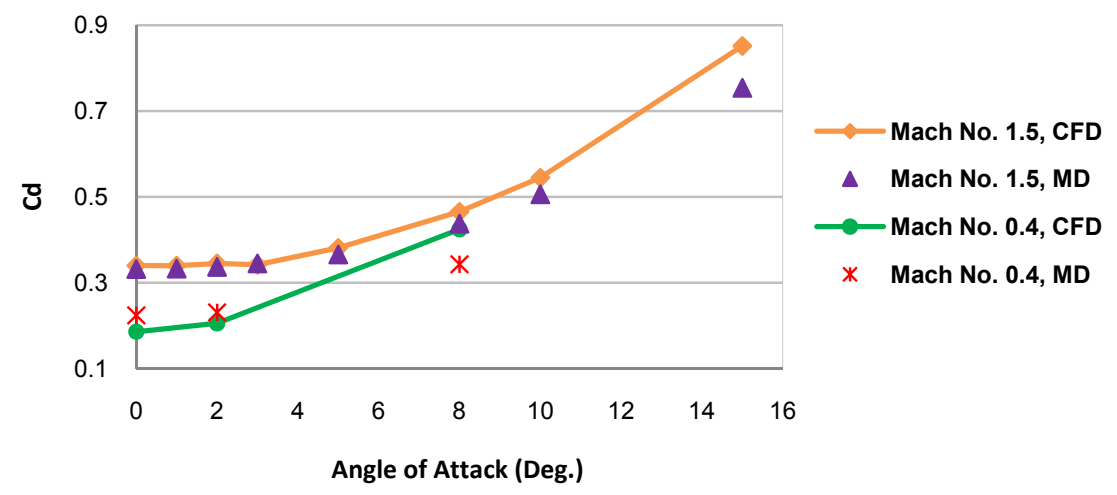

Figure 3: Comparison numerical and semi-experimental results of the drag coefficient against angles of attack before releasing drag surfaces. 


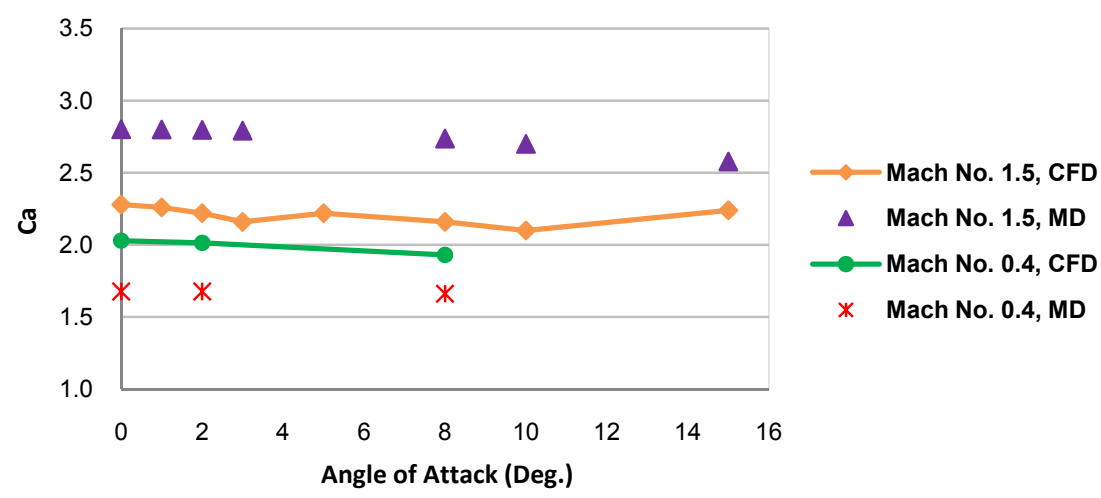

Figure 4: Comparison numerical and semi-experimental results of the axial force coefficient against angles of attack after releasing drag surfaces.

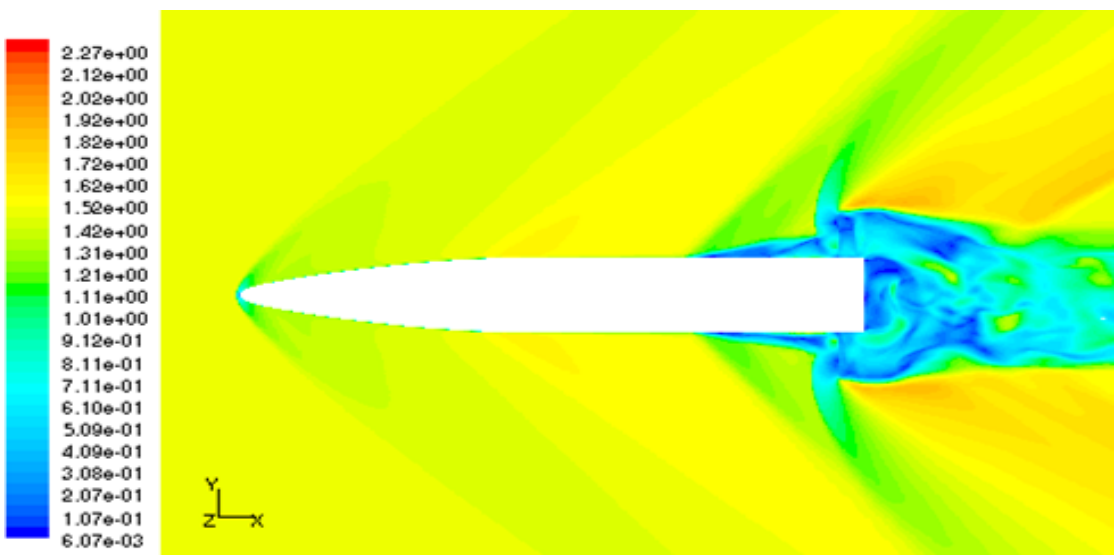

Figure 5: Mach number Contours after releasing drag surfaces, $\mathrm{M}=1.5$ at $\alpha=0^{\circ}$.

of the drag surfaces which has not been seen on body before releasing drag surfaces. The region of influence of the separation is seen to encompass a larger area of the body at supersonic flow as compared with the subsonic flow in figure 6 . Downstream of the drag surfaces, contra rotating vortices have been predicted which is shown in figure 7 in Mach number 0.4 and angle of attack 0 degree.

Since the surface area of body base is increased by releasing drag surfaces, it is expected the pressure base drag increased significantly. Eventually, the impingement upon the surface leads to changes in pressure distribution on the body surface which is shown in figure 8. This pressure distribution change results in different forces and moment on fins and body. Upstream of the drag surfaces, there is a pressure increase, which produces an existence of shock waves. 


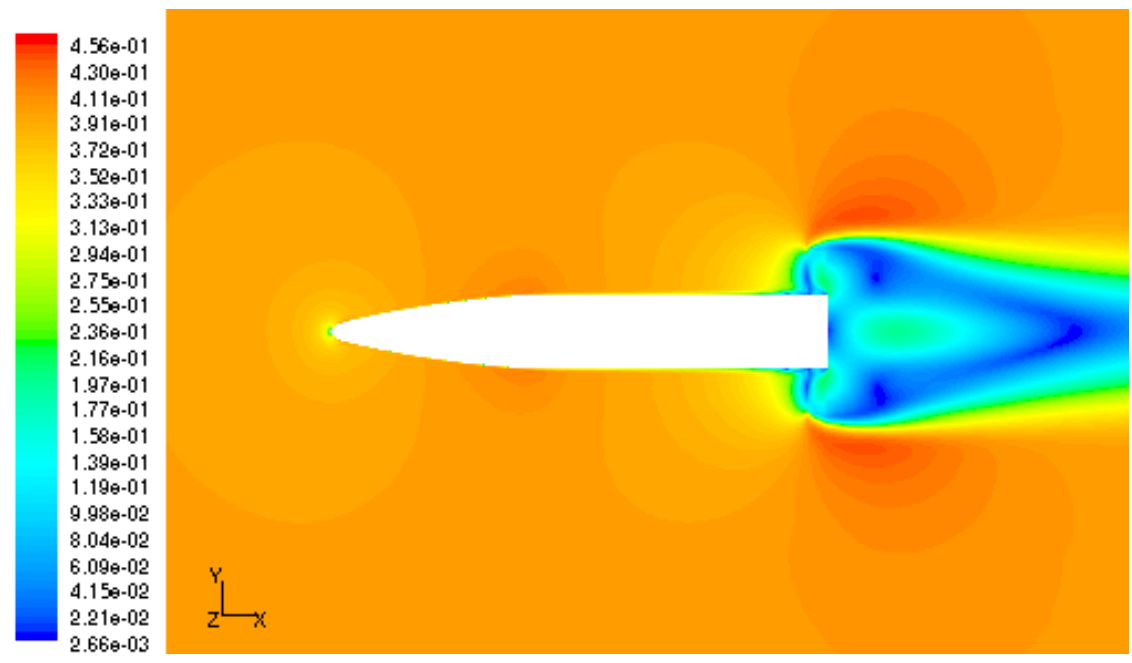

Figure 6: Mach number contours after releasing drag plates, $M=0.4$ at $\alpha=0^{\circ}$.

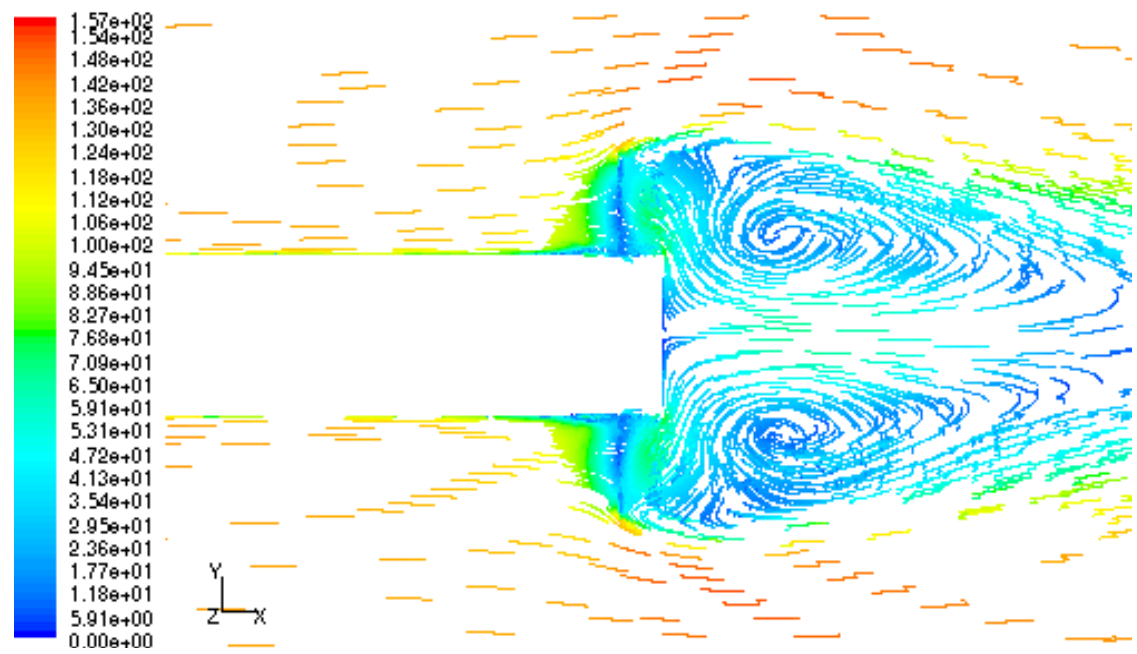

Figure 7: Side view of path line colored by velocity magnitude, Contra rotating vortices, $\mathrm{M}=0.4$ at $\alpha=0^{\circ}$.

A comparison between flow patterns on the body at 8 degrees angle of attack with 0 degree shows much more differences on flow. The first major difference between these cases and the zero incidence cases previously discussed is the surface area of the body affected by the drag surfaces. The boundary layer thickening occurs much earlier and the region of recirculation moves forward with increasing angle of attack on upper surface of body. The other regions of recirculation are those of the contra rotating vortices downstream of the drag surfaces. Whilst they appeared symmetric at zero incidences, they are asymmetric at an angle of attack. 


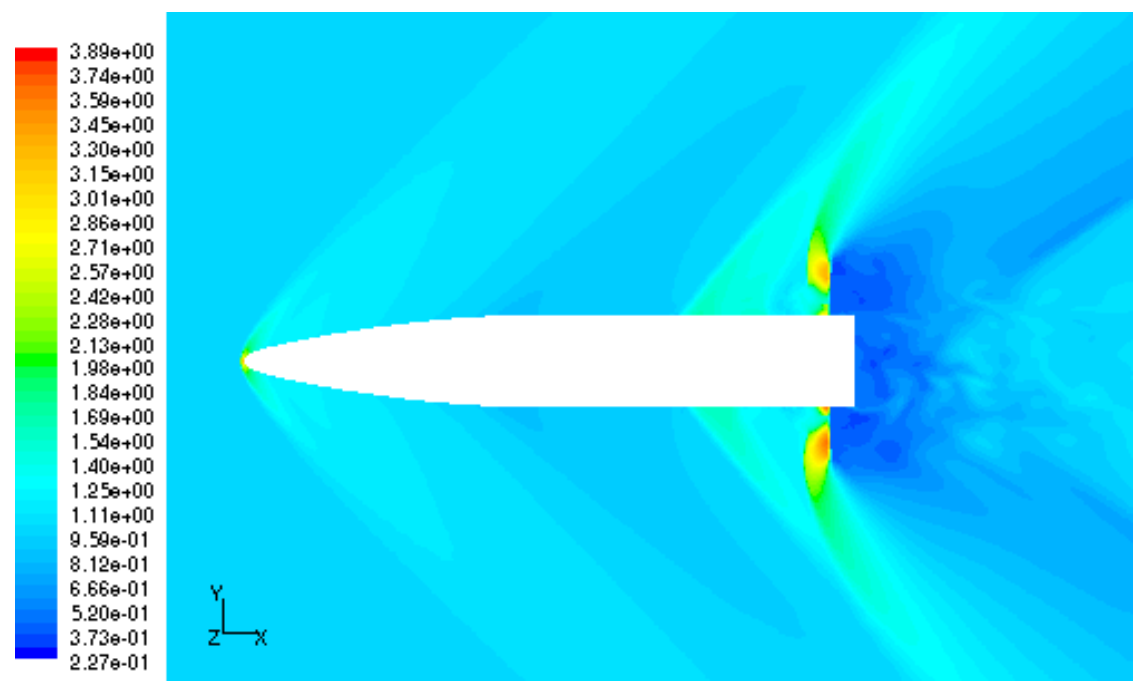

Figure 8: $\quad$ Static pressure contours after releasing drag plates, $M=1.5$ at $\alpha=0^{\circ}$ (atm).

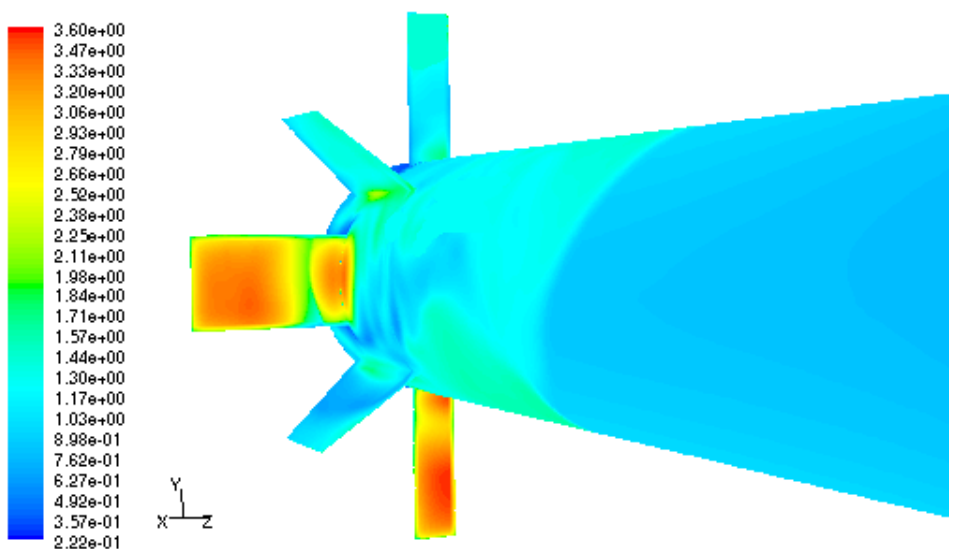

Figure 9: Static pressure contours after releasing drag plates, $M=1.5$ at $\alpha=8^{\circ}$ (atm).

In figure 9, the surface static pressure contours on one side of typical fin and drag surfaces are presented at Mach number 1.5 and 8 degrees of angle of attack. As can be seen, the difference flow patterns and difference pressure distribution on the opposing fin and drag surfaces produce a different load distribution. 


\subsection{Discussion}

A series of numerical models have been computed for a range of angles of attack in Mach numbers 1.5 and 0.4. Analysis of the aerodynamic coefficients provides understanding of how the drag plates affect the body aerodynamics. Figure 10 to 13 shows the change in aerodynamic coefficient versus angle of the attack for two models, before and after releasing drag surfaces.

Figures 10 and 11 show the change in drag coefficient versus angle of the attack for two models, before and after releasing drag surfaces. A comparison between coefficients shows that the significant change in drag force. In fact with releasing drag surfaces and placing them on deployed position increase complexity in flow and produce large vortices behind the base area. This complicated structure around the drag surfaces may consist of a strong secondary shock on body before facing fins and drag surfaces and interaction boundary layer of the fins and drag surfaces with shock waves. In addition second shock wave in supersonic region has a decrease in flow velocity and reverse flow on body. Consequence of this configuration of drag surfaces, the region of the dead flow behind the base of body increased which produce more pressure base drag. These phenomena cause the drag coefficient increased up to 10 times in $\mathrm{M}=0.4$ and 3 to 6 times in $\mathrm{M}=1.5$ at different angle of attack.

As shown in figures 12 and 13 the lift coefficient with increasing angle of attack decreased between 25 to 45 percent in $\mathrm{M}=1.5$ with different angles of attack and about 80 percent in $M=0.4$. This is due to the negative lift force on body which is produced by drag plates. In addition drag plates disturb the flow over its neighbouring fins which change pressure distribution on body and fins and reduce efficiency of the fins performance.

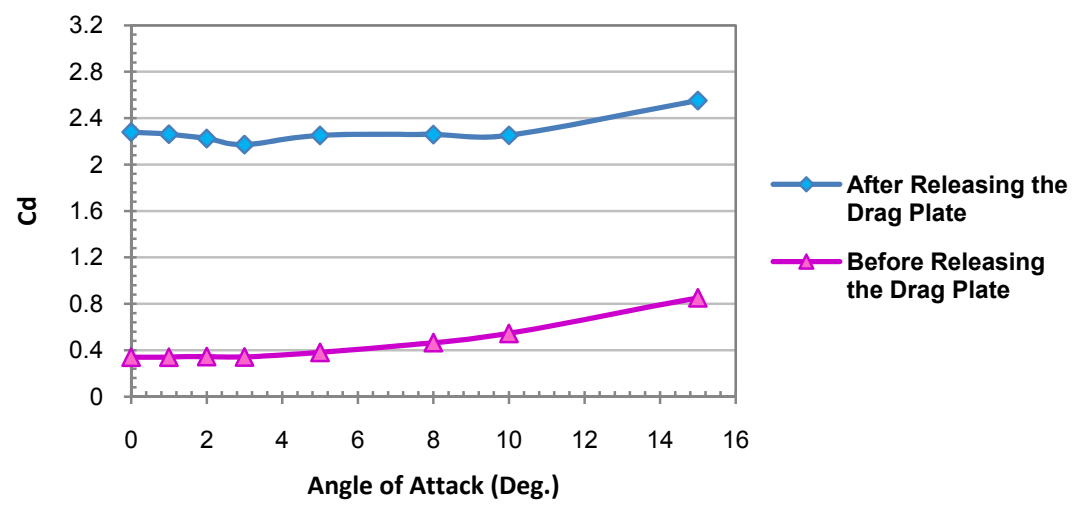

Figure 10: Comparison of the drag coefficient against angle of attack between two models, before and after releasing drag plates at $\mathrm{M}=1.5$. 


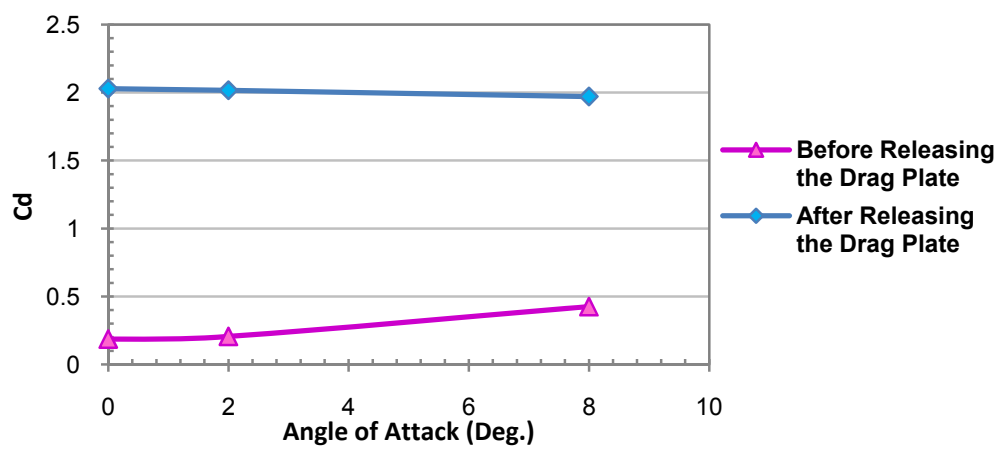

Figure 11: Comparison of the drag coefficient against angle of attack between two models, before and after releasing drag plates at $\mathrm{M}=0.4$.

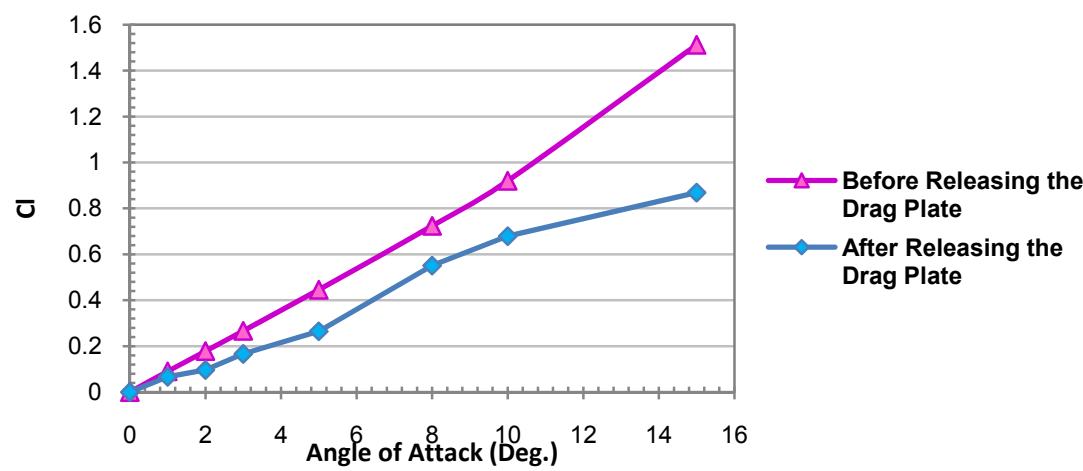

Figure 12: Comparison of the lift coefficient against angle of attack between two models, before and after releasing drag plates at $\mathrm{M}=1.5$.

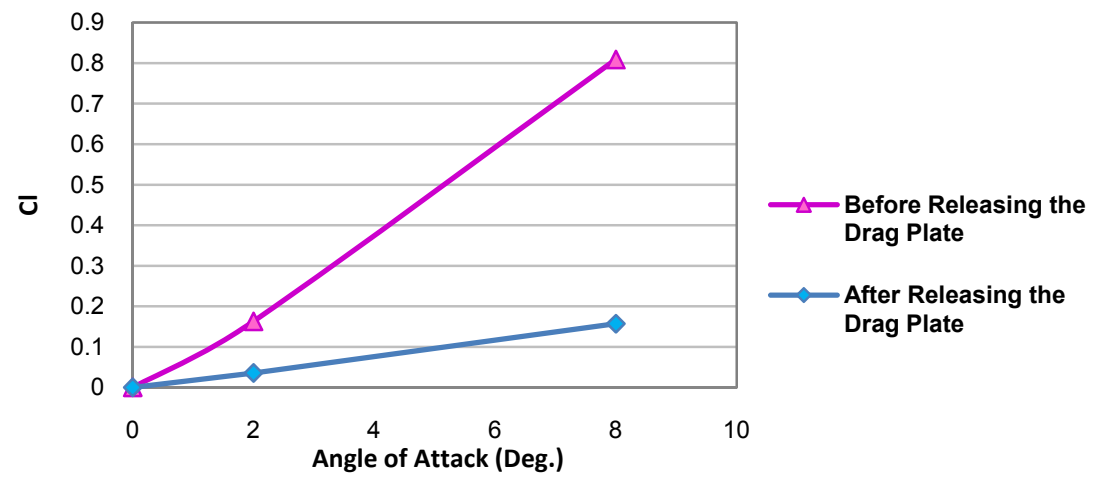

Figure 13: Comparison of the lift coefficient against angle of attack between two models, before and after releasing drag plates at $\mathrm{M}=0.4$. 


\section{Conclusion}

An investigation into the aerodynamic characteristics of a body with deployable drag surfaces is carried out. A series of numerical modelling is done for a range of angles of attack in Mach numbers 0.4 and 1.5 to include both subsonic and supersonic flow regimes. The study included two models of body with retracted position of drag surfaces and deployed position of drag surfaces. The drag and lift force coefficients were calculated from the flow field solutions. The results for retracted and deployed models were compared with each other in order to study the influence of drag surfaces on the aerodynamic force on the body.

A general study of the main aerodynamic coefficients shows that at all angles of attack, the coefficient of lift decreases and the coefficient of drag increases. This is due to the disturbing effect of the plates on the flow structure around the nearby fins which in its own turn decreases the performance of the set of fins significantly. The presence of the flat plate as local protuberance produces more drag force in different angles of attack as expected. This additional drag force brings body velocity and body rate of descent down to an acceptable level of speed for parachute deployment.

\section{References}

[1] Available Online: www.info-central.org/recovery techniques.shtml

[2] Anita Sengupta et al, Supersonic Performance of Disk-Gap-Band Parachutes Constrained to a 0-Degree Trim Angle, Journal of Spacecraft and Rockets, Vol. 46, No. 6, November-December 2009

[3] Richard Benneyet al, Aerodynamic Decelerator Systems-Diverse Challenges and Recent Advances, Journal of Aircraft, Vol. 38, No. 5, SeptemberOctober 2001

[4] Brandon P. Smith et al., A Historical Review of Inflatable Aerodynamic Decelerator Technology Development, IEEE Aerospace Conference, March, 2010

[5] Arash N. Lahouti et al., Design, Development and Testing of a Rigid Aerodynamic Decelerator System for Recovery Of a High-Altitude Sounding Rocket Payload, The 1st International ARA Days: Atmospheric Re-entry Systems, Missions and Vehicles, France, 2006

[6] Anon., Sounding Rocket Program Handbook, NASA Goddard Space Flight Center, Wallops Flight Facility, Wallops Island, Virginia, USA, June. 2005

[7] SRP-4 Design Team, "SRP-4 Design Document", University of Alaska Fairbanks, Alaska, USA, 2001

[8] Wm. David Washington et al., Experimental Investigation of Grid Fin Aerodynamics, Symposium on Missile Aerodynamics, Italy, 1998

[9] Mark Bell et al., A Numerical Study into a Local Protuberance Interaction with a Fin on Supersonic Projectile, 47th AIAA Aerospace Sciences Meeting Including the New Horizons Forum and Aerospace Exposition, Florida, 2009 